\title{
Iterative methods for zero points of accretive operators in Banach spaces
}

\author{
Sheng Hua Wang, Sun Young Cho, Xiao Long Qin
}

\begin{abstract}
The purpose of this paper is to consider the problem of approximating zero points of accretive operators. We introduce and analysis Mann-type iterative algorithm with errors and Halpern-type iterative algorithms with errors. Weak and strong convergence theorems are established in a real Banach space. As applications, we consider the problem of approximating a minimizer of a proper lower semicontinuous convex function in a real Hilbert space.
\end{abstract}

\section{Introduction-Preliminaries}

Let $C$ be a nonempty closed and convex subset of a Banach space $E$ and $E^{*}$ the dual space of $E$. Let $\langle\cdot, \cdot\rangle$ denote the pairing between $E$ and $E^{*}$. The normalized duality mapping $J: E \rightarrow 2^{E^{*}}$ is defined by

$$
J(x)=\left\{f \in E^{*}:\langle x, f\rangle=\|x\|^{2}=\|f\|^{2}\right\}
$$

for all $x \in E$. In the sequel, we use $j$ to denote the single-valued normalized duality mapping. Let $U=\{x \in E:\|x\|=1\}$. $E$ is said to be smooth or said to be have a Gâteaux differentiable norm if the limit

$$
\lim _{t \rightarrow 0} \frac{\|x+t y\|-\|x\|}{t}
$$

Key Words: Accretive operator; Fixed point; Nonexpansive mapping; Range condition; Zero point.

2010 Mathematics Subject Classification: Primary 47H05, 47H09; Secondary 47J25.

Received: November, 2010.

Accepted: February, 2012. 
exists for each $x, y \in U . E$ is said to have a uniformly Gâteaux differentiable norm if for each $y \in U$, the limit is attained uniformly for all $x \in U$. $E$ is said to be uniformly smooth or said to be have a uniformly Fréchet differentiable norm if the limit is attained uniformly for $x, y \in U$. It is known that if the norm of $E$ is uniformly Gâteaux differentiable, then the duality mapping $J$ is single valued and uniformly norm to weak* continuous on each bounded subset of $E$.

The modulus of convexity of $E$ is defined by

$$
\delta(\epsilon)=\inf \left\{1-\frac{\|x+y\|}{2}:\|x\| \leq 1,\|y\| \leq 1,\|x-y\| \geq \epsilon\right\}
$$

for every $\epsilon$ with $0 \leq \epsilon \leq 2$. A Banach space $E$ is said to be uniformly convex if $\delta(\epsilon)>0$ for every $\epsilon>0$. If $E$ is uniformly convex, then

$$
\left\|\frac{x+y}{2}\right\| \leq r\left(1-\delta\left(\frac{\epsilon}{r}\right)\right)
$$

for every $x, y \in E$ with $\|x\| \leq r,\|y\| \leq r$ and $\|x-y\| \geq \epsilon$.

In this paper, $\rightarrow$ and $\rightarrow$ denote strong and weak convergence, respectively.

A Banach space $E$ is said to satisfy Opial's condition [13] if for any sequence $\left\{x_{n}\right\} \subset E, x_{n} \rightarrow y$ implies that

$$
\liminf _{n \rightarrow \infty}\left\|x_{n}-y\right\|<\liminf _{n \rightarrow \infty}\left\|x_{n}-z\right\|
$$

for all $z \in E$ with $z \neq y$.

Recall that a mapping $T: C \rightarrow C$ is said to be nonexpanisve if

$$
\|T x-T y\| \leq\|x-y\|, \quad \forall x, y \in C .
$$

In this paper, we use $F(T)$ to denote the set of fixed points of $T$. A closed convex subset $C$ of $E$ is said to have the fixed point property for nonexpansive mappings if every nonexpansive mapping of a bounded closed convex subset $D$ of $C$ into itself has a fixed point in $D$.

A mapping $P$ of $C$ into itself is called a retraction if $P^{2}=P$. If a mapping $P$ of $C$ into itself is a retraction, then $P z=z$ for all $z \in R(P)$, where $R(P)$ is the range of $P$. A subset $D$ of $C$ is called a nonexpansive retract of $C$ if there exists a nonexpansive retraction from $C$ onto $D$.

Let $I$ denote the identity operator on $E$. An operator $A \subset E \times E$ with domain $D(A)=\{z \in E: A z \neq \emptyset\}$ and range $R(A)=\cup\{A z: z \in D(A)\}$ is said to be accretive if for each $x_{i} \in D(A)$ and $y_{i} \in A x_{i}, i=1,2$, there exists $j\left(x_{1}-x_{2}\right) \in J\left(x_{1}-x_{2}\right)$ such that

$$
\left\langle y_{1}-y_{2}, j\left(x_{1}-x_{2}\right)\right\rangle \geq 0 .
$$


An accretive operator $A$ is said to satisfy the range condition if

$$
\overline{D(A)} \subset \cap_{r>0} R(I+r A),
$$

where $\overline{D(A)}$ denote the closure of $D(A)$. An accretive operator $A$ is said to be $m$-accretive if $R(I+r A)=E$ for all $r>0$. In a real Hilbert space, an operator $A$ is $m$-accretive if and only if $A$ is maximal monotone.

For an accretive operator $A$, we can define a nonexpansive single-valued mapping $J_{r}: R(I+r A) \rightarrow D(A)$ by

$$
J_{r}=(I+r A)^{-1}
$$

for each $r>0$, which is called the resolvent of $A$. We also define the Yosida approximation $A_{r}$ by

$$
A_{r}=\frac{1}{r}\left(I-J_{r}\right) .
$$

It is known that $A_{r} x \in A J_{r} x$ for all $x \in R(I+r A)$ and $\left\|A_{r} x\right\| \leq \inf \{\|y\|: y \in$ $A x\}$ for all $x \in D(A) \cap R(I+r A)$.

One of classical methods of studying the problem $0 \in A x$, where $A \subset E \times E$ is an accretive operator, is the following:

$$
x_{0} \in E, \quad x_{n+1}=J_{r_{n}} x_{n}, \quad n \geq 0,
$$

where $J_{r_{n}}=\left(I+r_{n} A\right)^{-1}$ and $\left\{r_{n}\right\}$ is a sequence of positive real numbers.

The convergence of $(\Delta)$ has been studied by many authors; see, for example, Benavides, Acedo and Xu [1], Brézis and Lions [2], Bruck [3], Bruck and Passty [4], Bruck and Reich [5], Cho, Zhou and Kim [7], Ceng, Wu and Yao [8], Kamimur and Takahashi [10,11], Pazy [14], Qin, Kang and Cho [15], Qin and $\mathrm{Su}$ [16], Rockafellar [17], Reich [19-22], Takahashi and Ueda [23], Takahashi [24], $\mathrm{Xu}[26]$ and Zhou [27].

In this paper, motivated by the research work going on in this direction, we introduce and analysis Mann-type iterative algorithms with errors and Halpern-type iterative algorithms with errors. Weak and strong convergence theorems are established in a real Banach space.

In order to prove our main results, we need the following lemmas.

Lemma 1.1 ([21],[23]). Let $E$ be a real reflexive Banach space whose norm is uniformly Gâteaux differentiable and $A \subset E \times E$ be an accretive operator. Suppose that every weakly compact convex subset of $E$ has the fixed point property for nonexpansive mappings. Let $C$ be a nonempty, closed and convex subset of $E$ such that $\overline{D(A)} \subset C \subset \cap_{t>0} R(I+t A)$. If $A^{-1}(0) \neq \emptyset$, then the strong limit $\lim _{t \rightarrow \infty} J_{t} x$ exists and belongs to $A^{-1}(0)$ for all $x \in C$, where $J_{t}=(I+t A)^{-1}$ is the resolvent of $A$ for all $t>0$. 
Lemma $1.2([12])$. Let $\left\{a_{n}\right\},\left\{b_{n}\right\}$ and $\left\{c_{n}\right\}$ be three nonnegative real sequences satisfying

$$
a_{n+1} \leq\left(1-t_{n}\right) a_{n}+b_{n}+c_{n}, \quad n \geq 0,
$$

where $\left\{t_{n}\right\}$ is a sequence in $[0,1]$. Assume that the following conditions are satisfied

(a) $\sum_{n=0}^{\infty} t_{n}=\infty$ and $b_{n}=o\left(t_{n}\right)$;

(b) $\sum_{n=0}^{\infty} c_{n}<\infty$.

Then $\lim _{n \rightarrow \infty} a_{n}=0$.

Lemma $1.3([6])$. Let $C$ be a nonempty closed and convex subset of a uniformly convex Banach space $E$ and $T: C \rightarrow C$ a nonexpansive mapping. If a sequence $\left\{x_{n}\right\}$ in $C$ converges weakly to $z \in C$ and $\left\{x_{n}-T x_{n}\right\}$ converges strongly to 0 as $n \rightarrow \infty$, then $T z=z$.

Lemma $1.4([25])$. Let $\left\{a_{n}\right\}$ and $\left\{b_{n}\right\}$ be sequences of positive numbers satisfying

$$
a_{n+1} \leq a_{n}+b_{n}, \quad n \geq 0 .
$$

If $\sum_{n=0}^{\infty} b_{n}<\infty$, then the limit of $\left\{a_{n}\right\}$ exists.

Lemma 1.5 ([9]). In a Banach space $E$, there holds the inequality

$$
\|x+y\|^{2} \leq\|x\|^{2}+2\langle y, j(x+y)\rangle, \quad x, y \in E,
$$

where $j(x+y) \in J(x+y)$.

\section{Main results}

Theorem 2.1. Let $E$ be a real reflexive Banach space with a uniformly Gâteaux differentiable norm and $C$ a nonempty closed and convex subset of $E$. Let $P$ be a nonexpansive retraction of $E$ onto $C$ and $A \subset E \times E$ an accretive operator with $A^{-1}(0) \neq \emptyset$. Assume that $\overline{D(A)} \subset C \subset \cap_{r>0} R(I+r A)$. Let $\left\{x_{n}\right\}$ be a sequence generated by the following manner:

$$
x_{0} \in E, \quad x_{n+1}=\alpha_{n} u+\beta_{n} J_{r_{n}}\left(x_{n}+e_{n+1}\right)+\gamma_{n} P f_{n}, \quad n \geq 0,
$$

where $u \in C$ is a fixed point, $\left\{f_{n}\right\} \subset E$ is a bounded sequence, $\left\{\alpha_{n}\right\},\left\{\beta_{n}\right\}$ and $\left\{\gamma_{n}\right\}$ are sequences in $(0,1),\left\{e_{n}\right\}$ is a sequence in $E,\left\{r_{n}\right\} \subset(0, \infty)$ and $J_{r_{n}}=\left(I+r_{n} A\right)^{-1}$. Suppose that every weakly compact convex subset of $E$ has the fixed point property for nonexpansive mappings. Assume that the following conditions are satisfied 
(a) $\alpha_{n}+\beta_{n}+\gamma_{n}=1$;

(b) $\lim _{n \rightarrow \infty} \alpha_{n}=0$ and $\sum_{n=0}^{\infty} \alpha_{n}=\infty$;

(c) $\sum_{n=0}^{\infty} \gamma_{n}<\infty$ and $\sum_{n=1}^{\infty}\left\|e_{n}\right\|<\infty$;

(d) $r_{n} \rightarrow \infty$ as $n \rightarrow \infty$.

Then the sequence $\left\{x_{n}\right\}$ generated by $(\Upsilon)$ converges strongly to a zero of $A$.

Proof. First, we show that the sequence $\left\{x_{n}\right\}$ is bounded. Fixing $p \in A^{-1}(0)$, we have

$$
\begin{aligned}
\left\|x_{1}-p\right\| & =\left\|\alpha_{0} u+\beta_{0} J_{r_{0}}\left(x_{0}+e_{1}\right)+\gamma_{0} P f_{0}-p\right\| \\
& \leq \alpha_{0}\|u-p\|+\beta_{0}\left\|J_{r_{0}}\left(x_{0}+e_{1}\right)-p\right\|+\gamma_{0}\left\|P f_{0}-p\right\| \\
& \leq \alpha_{0}\|u-p\|+\beta_{0}\left\|\left(x_{0}+e_{1}\right)-p\right\|+\gamma_{0}\left\|f_{0}-p\right\| \\
& \leq \alpha_{0}\|u-p\|+\beta_{0}\left(\left\|x_{0}-p\right\|+\left\|e_{1}\right\|\right)+\gamma_{0}\left\|f_{0}-p\right\| \\
& \leq K
\end{aligned}
$$

where $K=\|u-p\|+\left\|x_{0}-p\right\|+\left\|e_{1}\right\|+\left\|f_{0}-p\right\|<\infty$. Putting

$$
M=\max \left\{K, \sup _{n \geq 0}\left\|f_{n}-p\right\|\right\},
$$

we prove that

$$
\left\|x_{n}-p\right\| \leq M+\sum_{i=1}^{n}\left\|e_{i}\right\|, \quad \forall n \geq 1 .
$$

It is easy to see that the result holds for $n=1$. We assume that the result holds for some $n$. It follows that

$$
\begin{aligned}
\left\|x_{n+1}-p\right\| & =\left\|\alpha_{n} u+\beta_{n} J_{r_{n}}\left(x_{n}+e_{n+1}\right)+\gamma_{n} P f_{n}-p\right\| \\
& \leq \alpha_{n}\|u-p\|+\beta_{n}\left\|J_{r_{n}}\left(x_{n}+e_{n+1}\right)-p\right\|+\gamma_{n}\left\|P f_{n}-p\right\| \\
& \leq \alpha_{n}\|u-p\|+\beta_{n}\left\|\left(x_{n}+e_{n+1}\right)-p\right\|+\gamma_{n}\left\|f_{n}-p\right\| \\
& \leq \alpha_{n}\|u-p\|+\beta_{n}\left\|x_{n}-p\right\|+\left\|e_{n+1}\right\|+\gamma_{n}\left\|f_{n}-p\right\| \\
& \leq \alpha_{n} M+\beta_{n}\left(M+\sum_{i=0}^{n}\left\|e_{i}\right\|\right)+\left\|e_{n+1}\right\|+\gamma_{n} M \\
& =M+\sum_{i=1}^{n+1}\left\|e_{i}\right\| .
\end{aligned}
$$

This shows that (2.1) holds. From the condition $\sum_{i=1}^{\infty}\left\|e_{i}\right\|<\infty$, we see that the sequence $\left\{x_{n}\right\}$ is bounded.

Next, we show that $\lim \sup _{n \rightarrow \infty}\left\langle u-z, J\left(x_{n+1}-z\right)\right\rangle \leq 0$, where $z=$ $\lim _{t \rightarrow \infty} J_{t} u$, which is guaranteed by Lemma 1.1. Note that $\frac{u-J_{t} u}{t} \in A J_{t} u$, $A_{r_{n}} x_{n} \in A J_{r_{n}} x_{n}$ and $A$ is accretive. It follows that

$$
\left\langle A_{r_{n}} x_{n}-\frac{u-J_{t} u}{t}, J\left(J_{r_{n}} x_{n}-J_{t} u\right)\right\rangle \geq 0 .
$$


This implies that

$$
\left\langle u-J_{t} u, J\left(J_{r_{n}} x_{n}-J_{t} u\right)\right\rangle \leq\left\langle t A_{r_{n}} x_{n}, J\left(J_{r_{n}} x_{n}-J_{t} u\right)\right\rangle .
$$

On the other hand, we have

$$
\lim _{n \rightarrow \infty}\left\|A_{r_{n}} x_{n}\right\|=\lim _{n \rightarrow \infty}\left\|\frac{x_{n}-J_{r_{n}} x_{n}}{r_{n}}\right\|=0
$$

In view of $(2.2)$, we arrive at

$$
\limsup _{n \rightarrow \infty}\left\langle u-J_{t} u, J\left(J_{r_{n}} x_{n}-J_{t} u\right)\right\rangle \leq 0, \quad \forall t \geq 0 .
$$

Since $z=\lim _{t \rightarrow \infty} J_{t} u$ and the norm of $E$ is uniformly Gâteaux differentiable, for any $\epsilon>0$, there exists $t_{0}>0$ such that

$$
\left|\left\langle z-J_{t} u, J\left(J_{r_{n}} x_{n}-J_{t} u\right)\right\rangle\right| \leq \frac{\epsilon}{2}
$$

and

$$
\left|\left\langle u-z, J\left(J_{r_{n}} x_{n}-J_{t} u\right)-J\left(J_{r_{n}} x_{n}-z\right)\right\rangle\right| \leq \frac{\epsilon}{2}
$$

for all $t \geq t_{0}$ and $n \geq 0$. It follows that

$$
\begin{aligned}
& \left|\left\langle u-J_{t} u, J\left(J_{r_{n}} x_{n}-J_{t} u\right)\right\rangle-\left\langle u-z, J\left(J_{r_{n}} x_{n}-z\right)\right\rangle\right| \\
\leq & \left|\left\langle u-J_{t} u, J\left(J_{r_{n}} x_{n}-J_{t} u\right)\right\rangle-\left\langle u-z, J\left(J_{r_{n}} x_{n}-J_{t} u\right)\right\rangle\right| \\
& +\left|\left\langle u-z, J\left(J_{r_{n}} x_{n}-J_{t} u\right)\right\rangle-\left\langle u-z, J\left(J_{r_{n}} x_{n}-z\right)\right\rangle\right| \\
= & \left|\left\langle z-J_{t} u, J\left(J_{r_{n}} x_{n}-J_{t} u\right)\right\rangle\right|+\left|\left\langle u-z, J\left(J_{r_{n}} x_{n}-J_{t} u\right)-J\left(J_{r_{n}} x_{n}-z\right)\right\rangle\right| \\
\leq & \epsilon
\end{aligned}
$$

for all $t \geq t_{0}$ and $n \geq 0$. It follows from (2.3) and (2.4) that

$$
\limsup _{n \rightarrow \infty}\left\langle u-z, J\left(J_{r_{n}} x_{n}-z\right)\right\rangle \leq \limsup _{n \rightarrow \infty}\left\langle u-J_{t} u, J\left(J_{r_{n}} x_{n}-J_{t} u\right)\right\rangle+\epsilon \leq \epsilon .
$$

Since $\epsilon$ is arbitrary, we see that

$$
\limsup _{n \rightarrow \infty}\left\langle u-z, J\left(J_{r_{n}} x_{n}-z\right)\right\rangle \leq 0
$$

Note that

$$
\left\|J_{r_{n}} x_{n}-J_{r_{n}}\left(x_{n}+e_{n+1}\right)\right\| \leq\left\|e_{n+1}\right\| .
$$

This implies that

$$
\lim _{n \rightarrow \infty}\left\|J_{r_{n}} x_{n}-J_{r_{n}}\left(x_{n}+e_{n+1}\right)\right\|=0 .
$$


Since $E$ has a uniformly Gâteaux differentiable norm, we arrive at

$$
\limsup _{n \rightarrow \infty}\left\langle u-z, J\left(J_{r_{n}}\left(x_{n}+e_{n+1}\right)-z\right)\right\rangle \leq 0 .
$$

On the other hand, , we see from the iterative $(\Upsilon)$ that

$x_{n+1}-J_{r_{n}}\left(x_{n}+e_{n+1}\right)=\alpha_{n}\left[u-J_{r_{n}}\left(x_{n}+e_{n+1}\right)\right]+\gamma_{n}\left[P f_{n}-J_{r_{n}}\left(x_{n}+e_{n+1}\right)\right]$.

That is,

$\left\|x_{n+1}-J_{r_{n}}\left(x_{n}+e_{n+1}\right)\right\| \leq \alpha_{n}\left\|u-J_{r_{n}}\left(x_{n}+e_{n+1}\right)\right\|+\gamma_{n}\left\|P f_{n}-J_{r_{n}}\left(x_{n}+e_{n+1}\right)\right\|$.

From the conditions (b) and (c), we obtain that

$$
\limsup _{n \rightarrow \infty}\left\|x_{n+1}-J_{r_{n}}\left(x_{n}+e_{n+1}\right)\right\|=0,
$$

which combines with (2.6) yields that

$$
\limsup _{n \rightarrow \infty}\left\langle u-z, J\left(x_{n+1}-z\right)\right\rangle \leq 0 .
$$

From the algorithm $(\Upsilon)$, we see that

$$
\begin{aligned}
x_{n+1}-z & =\alpha_{n}(u-z)+\beta_{n}\left[J_{r_{n}}\left(x_{n}+e_{n+1}\right)-z\right]+\gamma_{n}\left(P f_{n}-z\right) \\
& =\left(1-\alpha_{n}\right)\left[J_{r_{n}}\left(x_{n}+e_{n+1}\right)-z\right]+\alpha_{n}(u-z)+\gamma_{n}\left[P f_{n}-J_{r_{n}}\left(x_{n}+e_{n+1}\right)\right] .
\end{aligned}
$$

It follows from Lemma 1.5 that

$$
\begin{aligned}
& \left\|x_{n+1}-z\right\|^{2} \\
\leq & \left(1-\alpha_{n}\right)^{2}\left\|J_{r_{n}}\left(x_{n}+e_{n+1}\right)-z\right\|^{2}+2 \alpha_{n}\left\langle u-z, J\left(x_{n+1}-z\right)\right\rangle \\
& +2 \gamma_{n}\left\langle P f_{n}-J_{r_{n}}\left(x_{n}+e_{n+1}\right), J\left(x_{n+1}-z\right)\right\rangle \\
\leq & \left(1-\alpha_{n}\right)\left\|\left(x_{n}+e_{n+1}\right)-z\right\|^{2}+2 \alpha_{n}\left\langle u-z, J\left(x_{n+1}-z\right)\right\rangle \\
& +2 \gamma_{n}\left\|P f_{n}-J_{r_{n}}\left(x_{n}+e_{n+1}\right)\right\|\left\|x_{n+1}-z\right\| \\
\leq & \left(1-\alpha_{n}\right)\left(\left\|x_{n}-z\right\|^{2}-2\left\langle e_{n+1}, J\left[\left(x_{n}+e_{n+1}\right)-z\right]\right\rangle\right)+2 \alpha_{n}\left\langle u-z, J\left(x_{n+1}-z\right)\right\rangle \\
& +2 \gamma_{n}\left\|f_{n}-J_{r_{n}}\left(x_{n}+e_{n+1}\right)\right\|\left\|x_{n+1}-z\right\| \\
\leq & \left(1-\alpha_{n}\right)\left(\left\|x_{n}-z\right\|^{2}+2\left\|e_{n}+1\right\|\left\|\left(x_{n}+e_{n+1}\right)-z\right\|\right)+2 \alpha_{n}\left\langle u-z, J\left(x_{n+1}-z\right)\right\rangle \\
& +2 \gamma_{n}\left\|f_{n}-J_{r_{n}}\left(x_{n}+e_{n+1}\right)\right\|\left\|x_{n+1}-z\right\| \\
\leq & \left(1-\alpha_{n}\right)\left\|x_{n}-z\right\|^{2}+2 \alpha_{n}\left\langle u-z, J\left(x_{n+1}-z\right)\right\rangle \\
& +2 \gamma_{n}\left\|f_{n}-J_{r_{n}}\left(x_{n}+e_{n+1}\right)\right\|\left\|x_{n+1}-z\right\|+2\left\|e_{n+1}\right\|\left\|\left(x_{n}+e_{n+1}\right)-z\right\| \\
\leq & \left(1-\alpha_{n}\right)\left\|x_{n}-z\right\|^{2}+2 \alpha_{n}\left\langle u-z, J\left(x_{n+1}-z\right)\right\rangle+\left(\gamma_{n}+\left\|e_{n+1}\right\|\right) B,
\end{aligned}
$$

where $B$ is an appropriate constant such that

$B \geq \max \left\{\sup _{n \geq 0}\left\{2\left\|f_{n}-J_{r_{n}}\left(x_{n}+e_{n+1}\right)\right\|\left\|x_{n+1}-z\right\|\right\}, \sup _{n \geq 0}\left\{2\left\|\left(x_{n}+e_{n+1}\right)-z\right\|\right\}\right\}$ 
Let $\lambda_{n}=\max \left\{\left\langle u-z, J\left(x_{n+1}-z\right)\right\rangle, 0\right\}$. Next, we show that $\lim _{n \rightarrow \infty} \lambda_{n}=0$. Indeed, from (2.7), for any give $\epsilon>0$, there exists a positive integer $n_{1}$ such that

$$
\left\langle u-z, J\left(x_{n+1}-z\right)\right\rangle<\epsilon, \quad \forall n \geq n_{1} .
$$

This implies that $0 \leq \lambda_{n}<\epsilon \forall n \geq n_{1}$. Since $\epsilon>0$ is arbitrary, we see that $\lim _{n \rightarrow \infty} \lambda_{n}=0$. Put $a_{n}=\left\|x_{n}-z\right\|, b_{n}=2 \alpha_{n} \lambda_{n}, c_{n}=\left(\gamma_{n}+\left\|e_{n+1}\right\|\right) B$ and $t_{n}=\alpha_{n}$. In view of Lemma 1.2, we can obtain the desired conclusion immediately. This completes the proof.

In a real Hilbert space, Theorem 2.1 is reduced to the following.

Corollary 2.2. Let $H$ be a real Hilbert space and $C$ a nonempty, closed and convex subset of $H$. Let $P$ be a metric projection of $H$ onto $C$ and $A \subset H \times H$ a monotone operator with $A^{-1}(0) \neq \emptyset$. Assume that $\overline{D(A)} \subset C \subset$ $\cap_{r>0} R(I+r A)$. Let $\left\{x_{n}\right\}$ be a sequence generated by the following manner:

$$
x_{0} \in H, \quad x_{n+1}=\alpha_{n} u+\beta_{n} J_{r_{n}}\left(x_{n}+e_{n+1}\right)+\gamma_{n} P f_{n}, \quad n \geq 0,
$$

where $u \in C$ is a fixed point, $\left\{f_{n}\right\} \subset H$ is a bounded sequence, $\left\{\alpha_{n}\right\},\left\{\beta_{n}\right\}$ and $\left\{\gamma_{n}\right\}$ are sequences in $(0,1),\left\{e_{n}\right\}$ is a sequence in $H,\left\{r_{n}\right\} \subset(0, \infty)$ and $J_{r_{n}}=\left(I+r_{n} A\right)^{-1}$. Assume that the following conditions are satisfied

(a) $\alpha_{n}+\beta_{n}+\gamma_{n}=1$;

(b) $\lim _{n \rightarrow \infty} \alpha_{n}=0$ and $\sum_{n=0}^{\infty} \alpha_{n}=\infty$;

(c) $\sum_{n=0}^{\infty} \gamma_{n}<\infty$ and $\sum_{n=1}^{\infty}\left\|e_{n}\right\|<\infty$;

(d) $r_{n} \rightarrow \infty$ as $n \rightarrow \infty$.

Then the sequence $\left\{x_{n}\right\}$ converges strongly to a zero of $A$.

Theorem 2.3. Let $E$ be a real uniformly convex Banach space which satisfies Opial's condition and $C$ a nonempty closed and convex subset of $E$. Let $P$ be a nonexpansive retraction of $E$ onto $C$ and $A \subset E \times E$ an accretive operator with $A^{-1}(0) \neq \emptyset$. Assume that $\overline{D(A)} \subset C \subset \cap_{r>0} R(I+r A)$. Let $\left\{x_{n}\right\}$ be a sequence generated by the following manner:

$$
x_{0} \in C, \quad x_{n+1}=\alpha_{n} x_{n}+\beta_{n} J_{r_{n}}\left(x_{n}+e_{n+1}\right)+\gamma_{n} P f_{n}, \quad n \geq 0,
$$

where $\left\{f_{n}\right\} \subset E$ is a bounded sequence, $\left\{\alpha_{n}\right\},\left\{\beta_{n}\right\}$ and $\left\{\gamma_{n}\right\}$ are sequence in $(0,1),\left\{e_{n}\right\}$ is a sequence in $E,\left\{r_{n}\right\} \subset(0, \infty)$ and $J_{r_{n}}=\left(I+r_{n} A\right)^{-1}$. Assume that the following conditions are satisfied

(a) $\alpha_{n}+\beta_{n}+\gamma_{n}=1$; 
(b) $\lim \sup _{n \rightarrow \infty} \alpha_{n}<1$;

(c) $\sum_{n=0}^{\infty} \gamma_{n}<\infty$ and $\sum_{n=1}^{\infty}\left\|e_{n}\right\|<\infty$;

(d) $\liminf _{n \rightarrow \infty} r_{n}>0$.

Then the sequence $\left\{x_{n}\right\}$ generated by $(\Upsilon \Upsilon)$ converges weakly to a zero of $A$.

Proof. First, we show that the sequence $\left\{x_{n}\right\}$ is bounded. Fixing $p \in A^{-1}(0)$, we have

$$
\begin{aligned}
\left\|x_{1}-p\right\| & =\left\|\alpha_{0} x_{0}+\beta_{0} J_{r_{0}}\left(x_{0}+e_{1}\right)+\gamma_{0} P f_{0}-p\right\| \\
& \leq \alpha_{0}\left\|x_{0}-p\right\|+\beta_{0}\left\|J_{r_{0}}\left(x_{0}+e_{1}\right)-p\right\|+\gamma_{0}\left\|P f_{0}-p\right\| \\
& \leq \alpha_{0}\left\|x_{0}-p\right\|+\beta_{0}\left\|\left(x_{0}+e_{1}\right)-p\right\|+\gamma_{0}\left\|f_{0}-p\right\| \\
& \leq \alpha_{0}\left\|x_{0}-p\right\|+\beta_{0}\left(\left\|x_{0}-p\right\|+\left\|e_{1}\right\|\right)+\gamma_{0}\left\|f_{0}-p\right\| \\
& \leq K^{\prime}
\end{aligned}
$$

where $K^{\prime}=\left\|x_{0}-p\right\|+\left\|e_{1}\right\|+\left\|f_{0}-p\right\|<\infty$. Putting

$$
M^{\prime}=\max \left\{K, \sup _{n \geq 0}\left\|f_{n}-p\right\|\right\},
$$

we prove that

$$
\left\|x_{n}-p\right\| \leq M^{\prime}+\sum_{i=1}^{n}\left\|e_{i}\right\|, \quad \forall n \geq 1 .
$$

It is easy to see that the result holds for $n=1$. We assume that the result holds for some $n$. It follows that

$$
\begin{aligned}
\left\|x_{n+1}-p\right\| & =\left\|\alpha_{n} x_{n}+\beta_{n} J_{r_{n}}\left(x_{n}+e_{n+1}\right)+\gamma_{n} P f_{n}-p\right\| \\
& \leq \alpha_{n}\left\|x_{n}-p\right\|+\beta_{n}\left\|J_{r_{n}}\left(x_{n}+e_{n+1}\right)-p\right\|+\gamma_{n}\left\|P f_{n}-p\right\| \\
& \leq \alpha_{n}\left\|x_{n}-p\right\|+\beta_{n}\left\|\left(x_{n}+e_{n+1}\right)-p\right\|+\gamma_{n}\left\|f_{n}-p\right\| \\
& \leq \alpha_{n}\left\|x_{n}-p\right\|+\beta_{n}\left\|x_{n}-p\right\|+\left\|e_{n+1}\right\|+\gamma_{n}\left\|f_{n}-p\right\| \\
& \leq \alpha_{n} M+\beta_{n}\left(M+\sum_{i=0}^{n}\left\|e_{i}\right\|\right)+\left\|e_{n+1}\right\|+\gamma_{n} M \\
& =M+\sum_{i=1}^{n+1}\left\|e_{i}\right\| .
\end{aligned}
$$

This shows that (2.8) holds. From the condition $\sum_{i=1}^{\infty}\left\|e_{i}\right\|<\infty$, we see that the sequence $\left\{x_{n}\right\}$ is bounded.

Next, we show that $\lim _{n \rightarrow \infty}\left\|x_{n}-x^{*}\right\|$ exists for any $x^{*} \in A^{-1}(0)$. In fact, we have

$$
\begin{aligned}
\left\|x_{n+1}-x^{*}\right\| & =\left\|\alpha_{n} x_{n}+\beta_{n} J_{r_{n}}\left(x_{n}+e_{n+1}\right)+\gamma_{n} P f_{n}-x^{*}\right\| \\
& \leq \alpha_{n}\left\|x_{n}-x^{*}\right\|+\beta_{n}\left\|J_{r_{n}}\left(x_{n}+e_{n+1}\right)-x^{*}\right\|+\gamma_{n}\left\|P f_{n}-x^{*}\right\| \\
& \leq \alpha_{n}\left\|x_{n}-x^{*}\right\|+\beta_{n}\left\|\left(x_{n}+e_{n+1}\right)-x^{*}\right\|+\gamma_{n}\left\|f_{n}-x^{*}\right\| \\
& \leq\left\|x_{n}-x^{*}\right\|+\lambda_{n}
\end{aligned}
$$


where $\lambda_{n}=\left\|e_{n+1}\right\|+\gamma_{n}\left\|f_{n}-x^{*}\right\|$ for each $n \geq 0$. From the assumption, we see that $\sum_{n=0}^{\infty} \lambda_{n}<\infty$. It follows from Lemma 1.4 that $\lim _{n \rightarrow \infty}\left\|x_{n}-x^{*}\right\|$ exists for any $x^{*} \in A^{-1}(0)$. Put $d=\lim _{n \rightarrow \infty}\left\|x_{n}-x^{*}\right\|$ for any $x^{*} \in A^{-1}(0)$. We may, without loss of generality, assume that $d>0$. Since $A$ is accretive and $E$ is uniformly convex, we have

$$
\begin{aligned}
\left\|J_{r_{n}} x_{n}-x^{*}\right\| & \leq\left\|J_{r_{n}} x_{n}-x^{*}+\frac{r_{n}}{2}\left(A_{r_{n}} x_{n}-0\right)\right\| \\
& =\left\|J_{r_{n}} x_{n}-x^{*}+\frac{1}{2}\left(x_{n}-J_{r_{n}} x_{n}\right)\right\| \\
& =\left\|\frac{x_{n}+J_{r_{n}} x_{n}}{2}-x^{*}\right\| \\
& \leq\left\|x_{n}-x^{*}\right\|\left[1-\delta\left(\frac{\left\|x_{n}-J_{r_{n}} x_{n}\right\|}{\left\|x_{n}-x^{*}\right\|}\right)\right] .
\end{aligned}
$$

Note that

$$
\begin{aligned}
& \left\|x_{n+1}-x^{*}\right\| \\
& =\left\|\alpha_{n} x_{n}+\beta_{n} J_{r_{n}}\left(x_{n}+e_{n+1}\right)+\gamma_{n} P f_{n}-x^{*}\right\| \\
& \leq \alpha_{n}\left\|x_{n}-x^{*}\right\|+\beta_{n}\left\|J_{r_{n}}\left(x_{n}+e_{n+1}\right)-x^{*}\right\|+\gamma_{n}\left\|P f_{n}-x^{*}\right\| \\
& \leq \alpha_{n}\left\|x_{n}-x^{*}\right\|+\beta_{n}\left\|J_{r_{n}}\left(x_{n}+e_{n+1}\right)-J_{r_{n}} x_{n}\right\|+\beta_{n}\left\|J_{r_{n}} x_{n}-x^{*}\right\|+\gamma_{n}\left\|P f_{n}-x^{*}\right\| \\
& \leq \alpha_{n}\left\|x_{n}-x^{*}\right\|+\beta_{n}\left\|e_{n+1}\right\|+\beta_{n}\left\|J_{r_{n}} x_{n}-x^{*}\right\|+\gamma_{n}\left\|P f_{n}-x^{*}\right\| \\
& \leq \alpha_{n}\left\|x_{n}-x^{*}\right\|+\left\|e_{n+1}\right\|+\left(1-\alpha_{n}\right)\left\|J_{r_{n}} x_{n}-x^{*}\right\|+\gamma_{n}\left\|P f_{n}-x^{*}\right\| .
\end{aligned}
$$

This is,

$$
-\left(\alpha_{n}\left\|x_{n}-x^{*}\right\|+\left\|e_{n+1}\right\|+\left(1-\alpha_{n}\right)\left\|J_{r_{n}} x_{n}-x^{*}\right\|+\gamma_{n}\left\|P f_{n}-x^{*}\right\|\right) \leq-\left\|x_{n+1}-x^{*}\right\| .
$$

It follows from (2.9) and (2.10) that

$$
\begin{aligned}
& \left(1-\alpha_{n}\right)\left\|x_{n}-x^{*}\right\| \delta\left(\frac{\left\|x_{n}-J_{r_{n}} x_{n}\right\|}{\left\|x_{n}-x^{*}\right\|}\right) \\
& \leq\left(1-\alpha_{n}\right)\left(\left\|x_{n}-x^{*}\right\|-\left\|J_{r_{n}} x_{n}-x^{*}\right\|\right) \\
& =\left\|x_{n}-x^{*}\right\|-\left(\alpha_{n}\left\|x_{n}-x^{*}\right\|+\left(1-\alpha_{n}\right)\left\|J_{r_{n}} x_{n}-x^{*}\right\|\right) \\
& =\left\|x_{n}-x^{*}\right\|-\left(\alpha_{n}\left\|x_{n}-x^{*}\right\|+\left\|e_{n+1}\right\|+\left(1-\alpha_{n}\right)\left\|J_{r_{n}} x_{n}-x^{*}\right\|+\gamma_{n}\left\|P f_{n}-x^{*}\right\|\right) \\
& \quad+\left\|e_{n+1}\right\|+\gamma_{n}\left\|P f_{n}-x^{*}\right\| \\
& \leq\left\|x_{n}-x^{*}\right\|-\left\|x_{n+1}-x^{*}\right\|+\left\|e_{n+1}\right\|+\gamma_{n}\left\|P f_{n}-x^{*}\right\| .
\end{aligned}
$$

From the conditions (b), (c) and $\lim _{n \rightarrow \infty}\left\|x_{n}-x^{*}\right\|=d>0$, we arrive at

$$
\delta\left(\frac{\left\|x_{n}-J_{r_{n}} x_{n}\right\|}{\left\|x_{n}-x^{*}\right\|}\right) \rightarrow 0
$$

as $n \rightarrow \infty$. This implies that

$$
\lim _{n \rightarrow \infty}\left\|x_{n}-J_{r_{n}} x_{n}\right\|=0
$$


On the other hand, we have

$$
\begin{aligned}
\left\|J_{r_{n}} x_{n}-J_{1} J_{r_{n}} x_{n}\right\| & =\left\|\left(I-J_{1}\right) J_{r_{n}} x_{n}\right\| \\
& =\left\|A_{1} J_{r_{n}} x_{n}\right\| \\
& \leq \inf \left\{\|u\|: u \in A J_{r_{n}} x_{n}\right\} \\
& \leq\left\|A_{r_{n}} x_{n}\right\| \\
& =\left\|\frac{x_{n}-J_{r_{n}} x_{n}}{r_{n}}\right\| .
\end{aligned}
$$

From (2.11) and the condition (d), we obtain that

$$
\lim _{n \rightarrow \infty}\left\|J_{r_{n}} x_{n}-J_{1} J_{r_{n}} x_{n}\right\|=0
$$

Letting $v \in C$ be a weak subsequential limit of $\left\{x_{n}\right\}$ such that $x_{n_{i}} \rightarrow v$. From (2.11), we see that $J_{r_{n_{i}}} x_{n_{i}} \rightarrow v$. In view of Lemma 1.3, we obtain that $v \in F\left(J_{1}\right)=A^{-1}(0)$. Since the space satisfies Opial's condition (see [18]), we see that the desired conclusion holds. This completes the proof.

In a real Hilbert space, Theorem 2.3 is reduced to the following.

Corollary 2.4. Let $H$ be a real Hilbert space and $C$ a nonempty, closed and convex subset of $E$. Let $P$ be a metric projection of $E$ onto $C$ and $A \subset H \times H$ a monotone operator with $A^{-1}(0) \neq \emptyset$. Assume that $\overline{D(A)} \subset C \subset \cap_{r>0} R(I+$ $r A)$. Let $\left\{x_{n}\right\}$ be a sequence generated by the following manner:

$$
x_{0} \in C, \quad x_{n+1}=\alpha_{n} x_{n}+\beta_{n} J_{r_{n}}\left(x_{n}+e_{n+1}\right)+\gamma_{n} P f_{n}, \quad n \geq 0,
$$

where $\left\{f_{n}\right\} \subset H$ is a bounded sequence, $\left\{\alpha_{n}\right\},\left\{\beta_{n}\right\}$ and $\left\{\gamma_{n}\right\}$ are sequence in $(0,1),\left\{e_{n}\right\}$ is a sequence in $H,\left\{r_{n}\right\} \subset(0, \infty)$ and $J_{r_{n}}=\left(I+r_{n} A\right)^{-1}$. Assume that the following conditions are satisfied

(a) $\alpha_{n}+\beta_{n}+\gamma_{n}=1$;

(b) $\lim \sup _{n \rightarrow \infty} \alpha_{n}<1$;

(c) $\sum_{n=0}^{\infty} \gamma_{n}<\infty$ and $\sum_{n=1}^{\infty}\left\|e_{n}\right\|<\infty$;

(d) $\liminf _{n \rightarrow \infty} r_{n}>0$.

Then the sequence $\left\{x_{n}\right\}$ converges weakly to a zero of $A$.

\section{Applications}

In this section, as applications of main Theorems 2.1 and 2.3, we consider the problem of finding a minimizer of a convex function $f$. 
Let $H$ be a Hilbert space and $h: H \rightarrow(-\infty,+\infty]$ be a proper convex lower semi-continuous function. Then the subdifferential $\partial h$ of $h$ is defined as follows:

$$
\partial h(x)=\{y \in H: h(z) \geq h(x)+\langle z-x, y\rangle, \quad z \in H\}, \quad \forall x \in H .
$$

Theorem 3.1. Let $H$ be a real Hilbert space and $h: H \rightarrow(-\infty,+\infty] a$ proper convex lower semi-continuous function such that $\partial h(0) \neq \emptyset$. Let $\left\{x_{n}\right\}$ be a sequence generated by the following manner:

$$
\left\{\begin{array}{l}
x_{0} \in H \\
y_{n}=\arg \min _{x \in H}\left\{h(x)+\frac{1}{2 r_{n}}\left\|x-x_{n}-e_{n+1}\right\|^{2}\right\} \\
x_{n+1}=\alpha_{n} u+\beta_{n} y_{n}+\gamma_{n} f_{n}, \quad n \geq 0
\end{array}\right.
$$

where $u \in H$ is a fixed point, $\left\{f_{n}\right\} \subset H$ is a bounded sequence, $\left\{\alpha_{n}\right\},\left\{\beta_{n}\right\}$ and $\left\{\gamma_{n}\right\}$ are sequences in $(0,1),\left\{e_{n}\right\}$ is a sequence in $H$ and $\left\{r_{n}\right\} \subset(0, \infty)$. Assume that the following conditions are satisfied

(a) $\alpha_{n}+\beta_{n}+\gamma_{n}=1$;

(b) $\lim _{n \rightarrow \infty} \alpha_{n}=0$ and $\sum_{n=0}^{\infty} \alpha_{n}=\infty$;

(c) $\sum_{n=0}^{\infty} \gamma_{n}<\infty$ and $\sum_{n=1}^{\infty}\left\|e_{n}\right\|<\infty$;

(d) $r_{n} \rightarrow \infty$ as $n \rightarrow \infty$.

Then the sequence $\left\{x_{n}\right\}$ converges strongly to a minimizer of $h$.

Proof. Since $h: H \rightarrow(-\infty,+\infty]$ is a proper convex lower semi-continuous function, we have that the subdifferential $\partial h$ of $h$ is maximal monotone by Rockafellar [18]. Notice that

$$
y_{n}=\arg \min _{x \in H}\left\{h(x)+\frac{1}{2 r_{n}}\left\|x-x_{n}-e_{n+1}\right\|^{2}\right\}
$$

is equivalent to the following

$$
0 \in \partial h\left(y_{n}\right)+\frac{1}{r_{n}}\left(y_{n}-x_{n}-e_{n+1}\right) .
$$

It follows that

$$
x_{n}+e_{n+1} \in y_{n}+r_{n} \partial h\left(y_{n}\right), \quad \forall n \geq 0 .
$$

By Theorem 2.1, we can obtain the desired conclusion immediately. 
Theorem 3.2. Let $H$ be a real Hilbert space and $h: H \rightarrow(-\infty,+\infty] a$ proper convex lower semi-continuous function such that $\partial h(0) \neq \emptyset$. Let $\left\{x_{n}\right\}$ be a sequence generated by the following manner:

$$
\left\{\begin{array}{l}
x_{0} \in H, \\
y_{n}=\arg \min _{x \in H}\left\{h(x)+\frac{1}{2 r_{n}}\left\|x-x_{n}-e_{n+1}\right\|^{2}\right\}, \\
x_{n+1}=\alpha_{n} x_{n}+\beta_{n} y_{n}+\gamma_{n} f_{n}, \quad n \geq 0,
\end{array}\right.
$$

where $\left\{f_{n}\right\} \subset H$ is a bounded sequence, $\left\{\alpha_{n}\right\},\left\{\beta_{n}\right\}$ and $\left\{\gamma_{n}\right\}$ are sequence in $(0,1),\left\{e_{n}\right\}$ is a sequence in $H$ and $\left\{r_{n}\right\} \subset(0, \infty)$. Assume that the following conditions are satisfied

(a) $\alpha_{n}+\beta_{n}+\gamma_{n}=1$;

(b) $\lim \sup _{n \rightarrow \infty} \alpha_{n}<1$;

(c) $\sum_{n=0}^{\infty} \gamma_{n}<\infty$ and $\sum_{n=1}^{\infty}\left\|e_{n}\right\|<\infty$;

(d) $\liminf _{n \rightarrow \infty} r_{n}>0$.

Then the sequence $\left\{x_{n}\right\}$ converges weakly to a minimizer of $h$.

Proof. We can easily obtain from the proof of Theorem 2.3 and Theorem 3.1 the desired conclusion.

\section{Acknowledgement}

The third author was supported by Natural Science Foundation of Zhejiang Province (Q12A010097), and National Natural Science Foundation of China (11126334).

\section{References}

[1] T.D. Benavides, G.L. Acedo, H.K. Xu, Iterative solutions for zeros of accretive operators, Math. Nachr. 248 (2003) 62-71.

[2] H. Brézis, P.L. Lions, Produits infinis de resolvants, Israel J. Math. 29 (1978) 329-345.

[3] R.E. Bruck, A strongly convergent iterative method for the solution of $0 \in U x$ for a maximal monotone operator $U$ in Hilbert space, J. Math. Appl. Anal. 48 (1974) 114-126.

[4] R.E. Bruck, G.B. Passty, Almost convergence of the infinite product of resolvents in Banach spaces, Nonlinear Anal. 3 (1979) 279-282. 
[5] R.E. Bruck, S. Reich, Nonlinear projections and resolvents of accretive operators in Banach spaces, Houston. J. Math. 3 (1977) 459-470.

[6] F.E. Browder, Semicontractive and semiaccretive nonlinear mappings, in Banach spaces, Bull. Amer. Math. Soc. 74 (1968) 660-665.

[7] Y.J. Cho, H. Zhou, J.K. Kim, Iterative approximations of zeros for accretive operators in Banach spaces, Commun. Korean. Math. Soc. 21 (2006) 237-251.

[8] L.C. Ceng, S.Y. Wu, J.C. Yao, New accuracy criteria for modified approximate proximal point algorithms in Hilbert spaces, Taiwanese J. Math. 12 (2008) 1691-1705.

[9] J.S. Jung, Y.J. Cho, H. Zhou, Iterative processes with mixed errors for nonlinear equations with perturbed $m$-accretive operators in Banach spaces, Appl. Math. Comput. 133 (2002) 389-406.

[10] S. Kamimura, W. Takahashi, Weak and strong convergence of solutions to accretive operator inclusions and Applications, Set-Valued Anal. 8 (2000) 361-374.

[11] S. Kamimura, W. Takahashi, Approximating solutions of maximal monotone operators in Hilbert spaces, J. Approx. Theory, 106 (2000) 226-240.

[12] L.S. Liu, Ishikawa and Mann iterative process with errors for nonlinear strongly accretive mappings in Banach spaces, J. Math. Anal. Appl. 194 (1995) 114-125.

[13] Z. Opial, Weak convergence of the sequence of successive a pproximations for nonexpansive mappings, Bull. Amer. Math. Soc. 73 (1967) 591-597.

[14] A. Pazzy, Remarks on nonlinear ergodic theory in Hilbert space, Nonlinear Anal. 6 (1979) 863-871.

[15] X. Qin, S.M. Kang, Y.J. Cho, Approximating zeros of monotone operators by proximal point algorithms, J. Glob. Optim. 46 (2010) 75-87.

[16] X. Qin, Y. Su, Approximation of a zero point of accretive operator in Banach spaces, J. Math. Anal. Appl. 329 (2007) 415-424.

[17] R.T. Rockafellar, Monotone operators and the proximal point algorithm, SIAM J. Control Optim. 14 (1976) 877-898.

[18] R.T. Rockafellar, Characterization of the subdifferentials of convex functions, Pacific J. Math. 17 (1966) 497-510. 
[19] S. Reich, On infinite products of resolvents, Atti Acad. Naz Lincei 63 (1977) 338-340.

[20] S. Reich, Weak convergence theorems for resolvents of accretive operators in Banach space, J. Math. Anal. Appl. 67 (1979) 274-276.

[21] S. Reich, Strong convergence theorems for resolvents of accretive operators in Banach spaces, J. Math. Anal. Appl. 75 (1980) 287-292.

[22] S. Reich, Constructing zeros of accretive operators, Appl. Anal. 8 (1979) 349-352.

[23] W. Takahashi, Y. Ueda, On Reich's strong convergence theorems for resolvents of accretive operators, J. Math. Anal. Appl. 104 (1984) 546-553.

[24] W. Takahashi, Viscosity approximation methods for resolvents of acretive operators in Banach space, J. Fixed Point Theory Appl. 1 (2007) 135-147.

[25] K.K. Tan, H.K. Xu, Approximating fixed points of nonexpansive mappings by the Ishikawa iteration process, J. Math. Anal. Appl. 178 (1993) 301-308.

[26] H.K. Xu, Iterative algorithms for nonlinear operators, J. London Math. Soc. 66 (2002) 240-256.

[27] H. Zhou, Remarks on the approximation methods for nonlinear operator equations of the $m$-accretive type, Nonlinear Anal. 42 (2000) 63-69.

Sheng Hua Wang,

Department of Mathematics and Physics,

North China Electric Power University,

Baoding 071003, China.

Email: sheng-huawang@hotmail.com

Sun Young Cho,

Department of Mathematics,

Gyeongsang National University,

Jinju 660-701, Korea.

Email: ooly61@yahoo.co.kr

Xiao Long Qin,

Department of Mathematics,

Hangzhou Normal University,

Hangzhou 310036, China.

Email: qxlxajh@163.com 
